\title{
ROBUST DROWN ALERTING, PREVENTING AND AUTONOMOUS RESCUE SYSTEM
}

\author{
Mr. PILLALAMARRI LAXMAN ${ }^{1}$, prof. ANUJ JAIN ${ }^{2}$ \\ ${ }^{1}$ LPU.IN Ph.D SCHOLAR (41800281), ${ }^{2}$ Lovely Professional University, India \\ E-mail: laxmanabcd@gmail.com, anuj.22631@lpu.co.in
}

\begin{abstract}
This paper introduces a novel method of automatic life-saving mechanism to save a drowning victim in a helpless condition. The realization of this method may be done by an Arduino Board or an embedded microcontroller. This method utilizes artificial intelligence to save drowning victims from fatal death and rings a loudspeaker.
\end{abstract}

Keywords: swimming pool, drowning accident, drowning detection system, drowning prevention system, waterside monitoring system, drowning detection, portable bather monitoring device, responsive swimming pool, drowning victim, swimming pool safety

\section{LITERATURE STUDY}

Drowning is a silent process where a victim cannot shout for help because of water blocking mouth. As soon as the drowning victim drinks enough water and gains weight and reaches the swimming pool floor, the victim's body will settle on the floor due to gravity. After several hours, microorganisms in the stomach and lungs will produce and the dead body will float on water. This floating may take several hours to several days based on water conditions. If the drowned person is taken out of the surface within five or six minutes, survival chances were more without severe brain or organ damage.

A. Roy [1], introduced wearable swimming goggles housing a buzzer and hydrophone backed by an embedded software program. J. Geetha Ramani [2], came up with a solution to this drowning problem by wearable wristband with an inflatable balloon hidden inside a casing, able to work automatically or by the manual trigger. S. Sindhuja [5], has an idea of integrating pressure sensors, GPS to send SMS alert messages, housing an airbag either automatically or by a manual trigger mechanism. Muhammad Ramdhan MS1, Muhammad Ali [7] come up with a headband type model with IoT technology integrated by several sensors like a pulse-oxytomertic sensor, RF Signal Detector, GPS for SMS alert. Y. Nishida [9] came up with an idea of leaving a spherical transparent device housing a gyroscope, motion sensor to identify the event of a baby falling in a bathtub or any other small water body indoors. M. A. Hayat [3], [6] Yaswanth Kumar S K; Praveen O K, CAI Xiaoyang [10], L. Fei, W. Xueli [11], Soren Bonderup [12], [14] Ahmad Ilham, Sherly Prastica Della have proposed several methods by using image processing techniques to predict, detect and avoid drowning. A. Kulkarni [8] has proposed an embedded device housing several sensors to detect a drowning incident [4], [13].

\section{PROPOSED METHOD}

After a thorough examination of the prior art, proposing a novel autonomous rescue system for swimming pools [19]. Arduino Uno board, an on-chip CPU is used to sense signals and instruct the other electrical components in the system. Main electrical components include screw jack driver motors and buzzer. Figure 1 shows the main idea of the proposed system.

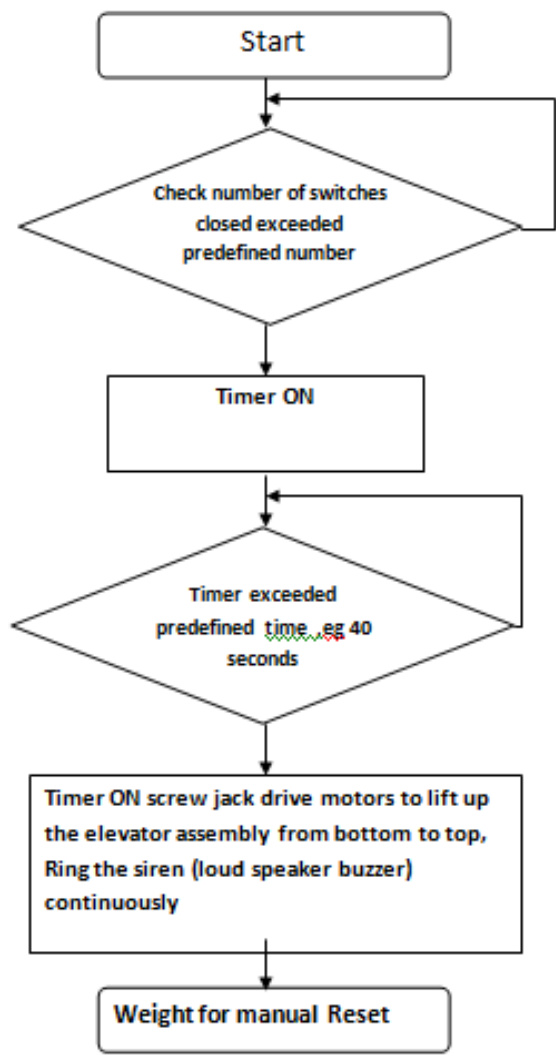

Figure 1. Block Diagram of proposed system

In the proposed method, a swimming pool prototype is prepared and a switch matrix is arranged on the bottom of the swimming pool floor, a "Mechanical Lifting and Pulling Screw Jacks floor (elevator assembly)" (Figure 2 and 3) is placed on the switch matrix. The "Mechanical Lifting and Pulling Screw Jacks floor (elevator assembly)" is having small circular equal-spaced gaps areas where switches arranged in the bottom elevator assembly are covered by a smooth latex-like rubber sheet is arranged. If any person lies down after drown on the Smooth rubber sheet bump, presses the switch under the rubber sheet 
bump (trigger button) due to the weight of the drowning body eventually triggers the "Mechanical Lifting and Pulling Screw Jacks floor (elevator assembly)" by high torque motors controlled by CPU leads to the automatic lifting of person who is struggling/unconscious on swimming pool bottom floor, after a threshold time set by the programmer. The threshold time is close to 40 to 60 seconds.

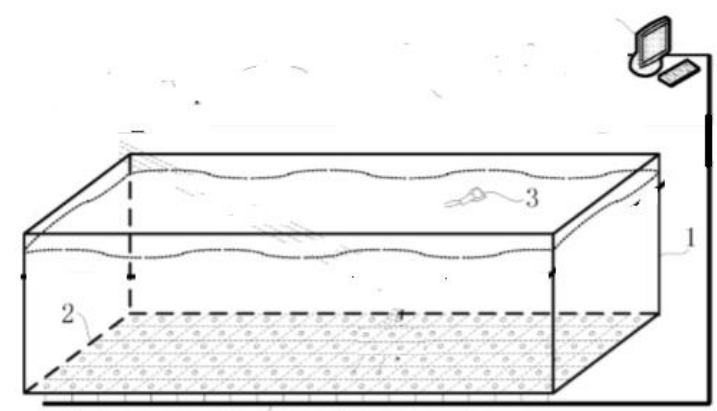

Figure 2. Proposed Swimming pool with switches arranged all places on bottom and connected to CPU.

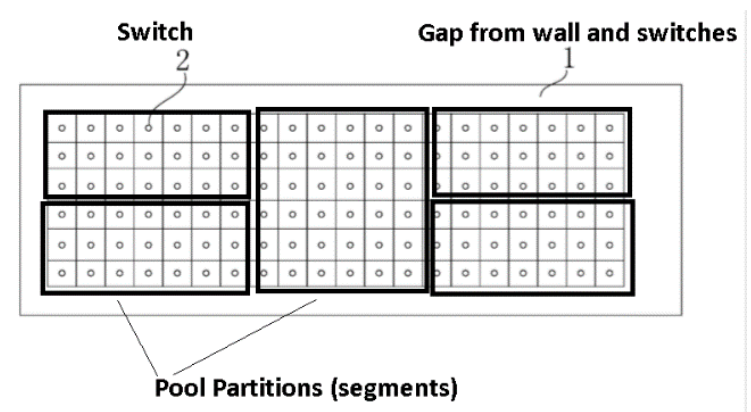

Figure 3. Swimming pool (top view) segmentation various styles based on shape and size of swimming pool.

As soon as drowning is detected, a siren should be ringing at the same time. This will increase the water level of the drowning victim and speed up the surfacing of the body.

The proposed system measures the amount of time a body lies down on the bottom and lifts the body after a preset threshold time which will eventually prevent death.

\section{TACTICAL SWITCHES}

Standard Tactile Switches consist of four components: cover, plunger, contact dome and base.

The base combines the contacts and terminals into a single component.

Every switch is monitored continuously by the CPU board every 10 seconds. The type of trigger switches is shown in fig 4 and 5.

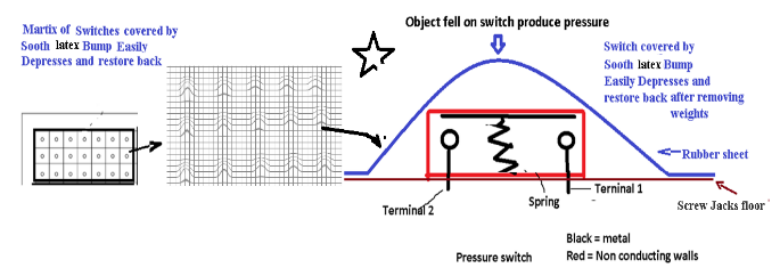

Figure 4. CPU with switch mechanism without swimming pool prototype with CPU and PUSH BUTTON.
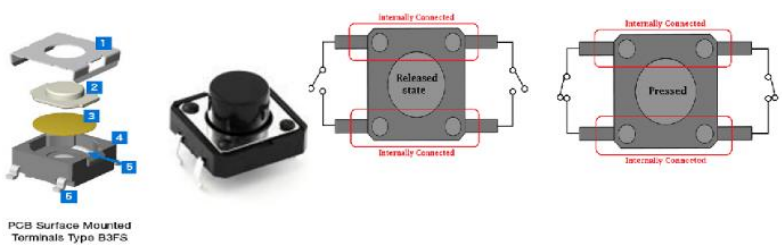

Figure 5. Illustration of tactile switch with detailed explanation.

Once a switch press is detected, the CPU will start counting the number of switches pressed. If the number of switches pressed continuously by an external force is more than the preset threshold number in that segment, then the CPU will wait for 50 seconds. Even after 50 seconds, still, the number of switches pressed are not changing in that segment, the CPU assumes a person is unconscious on the swimming pool floor, then a non-mask-able command is generated to turns $\mathrm{ON}$ the motor corresponding to the respective segment to lift the "Mechanical Lifting and Pulling Screw Jacks floor(elevator assembly)" along with anything lying on the "Mechanical Lifting and Pulling Screw Jacks floor(elevator assembly)" assuming as lifting the drowning victim. As soon as the drown victim assumed as out of the water, the CPU will wait for the RESET command manually. Whenever a RESET command given by pressing the RESET button on CPU, The "Mechanical Lifting and Pulling Screw Jacks floor (elevator assembly)" mechanism will go back to the bottom of the swimming pool and water will enter the swimming pool normally. After this RESET mechanism again CPU starts checking for any switch press and cycle repeats as shown in Figure 1 .

In this method, the swimming pool is divided into a single segment or several segments and several electrical toggle switches were placed and connected parallel to the CPU. Each segment is covered by a "Mechanical Lifting and Pulling Screw Jacks floor or elevator assembly" attached to individual motors which can easily lift to the top of the pool as shown in Figure 6. The liftable floor (elevator assembly) is having smooth rubber areas where any pressure will turn ON the switches which are exactly under the smooth areas. These smooth rubber areas were arranged a little bump like structure (trigger points) so that anyone standing on the bump (trigger) will immediately detect a switch press and triggers the motors and moves "Mechanical Lifting and Pulling Screw Jacks floor (elevator assembly)" upwards as shown in Figure 6. 


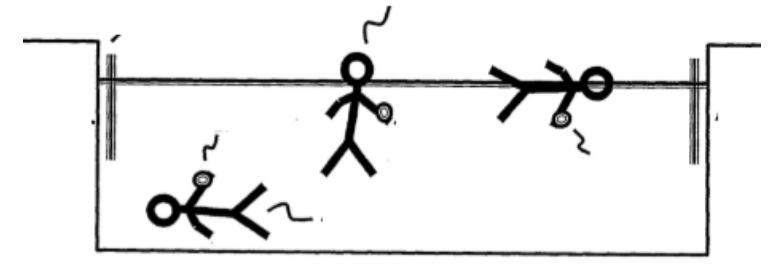

Figure 6.a. Showing person at the bottom (victim) pressing switches on the pool floor, and a safe person close to the surface.

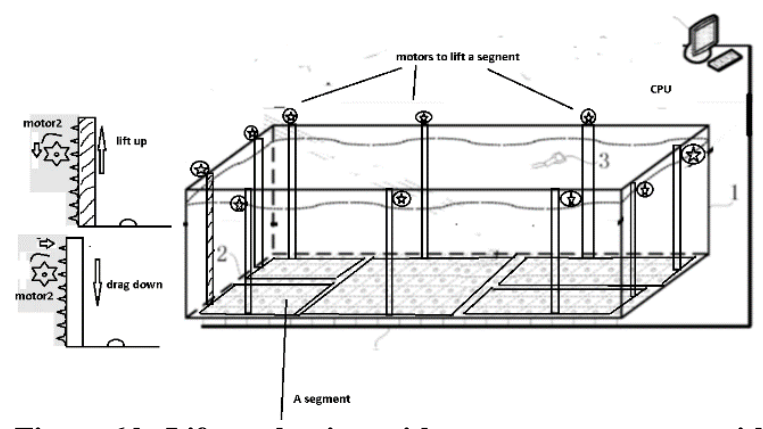

Figure 6.b. Lift mechanism with motor arrangement with "Mechanical Lifting and Pulling Screw Jacks floor (elevator assembly)" Screw jack Mechanism.

The management of the swimming pool should place a signboard about this system and warn the swimmers not to step on triggers more than 30 seconds accidentally to avoid false alarms.

The above system is a special mechanism to easily quickly lift anything in the machine with a single motor efficiently with the help of a Screw jack is shown in Figure 7.

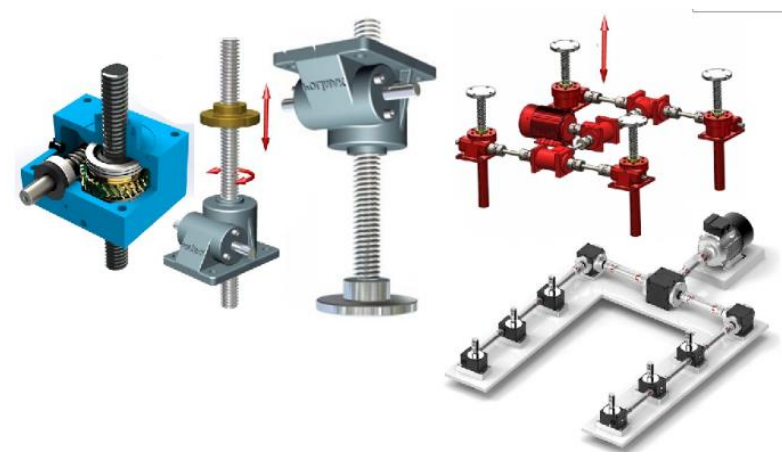

Figure 7. "Mechanical Lifting and Pulling Screw Jacks floor (elevator assembly)" Screw jack Mechanism working (two arrangements) with a single motor.

All the segments will rest on the top surface once automatically by the computer instructions the swimming pool is marked OFF at night times and in the times there are no adults or not in use. This will prevent kids or pets from falling in the pool.

\section{SCREW JACK MECHANISM STUDY}

A screw jack is used in applications where linear motion is required, as shown in Figure 8. Lifting of any load, pushing, or pulling of mechanical equipment, adjusting of tight clearances of mechanical parts can be done by screw jacks. The mechanical capacity of screw jacks is between $5 \mathrm{kN}$ and $2000 \mathrm{kN}$. Jack screws can be used as linear motors, linear actuators, or mechanical lifts depending on the type of motion. They can be employed to lift, pull, move, or align any kind of loads, with perfect synchronism which can hardly be obtained with other handling methods.

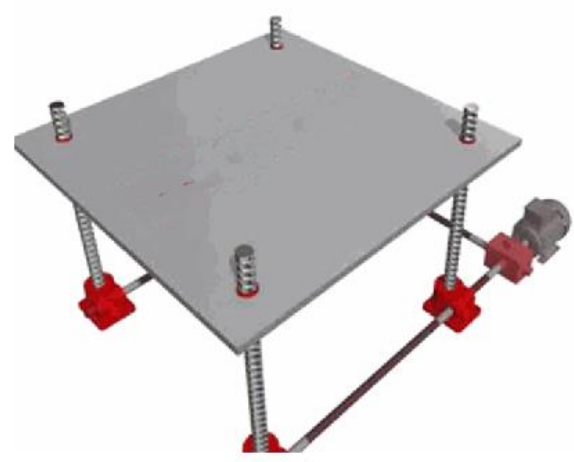

Figure 8. The above figure illustrates how a single motor lifts a plane equally without imbalance linearly.

"POWER JACKS" Equipment manufacturer (www.powerjacks.com) [18], has given a scientific formula to calculate speed, torque, and power of uniform elevator assembly supported by screw-jack as follows.

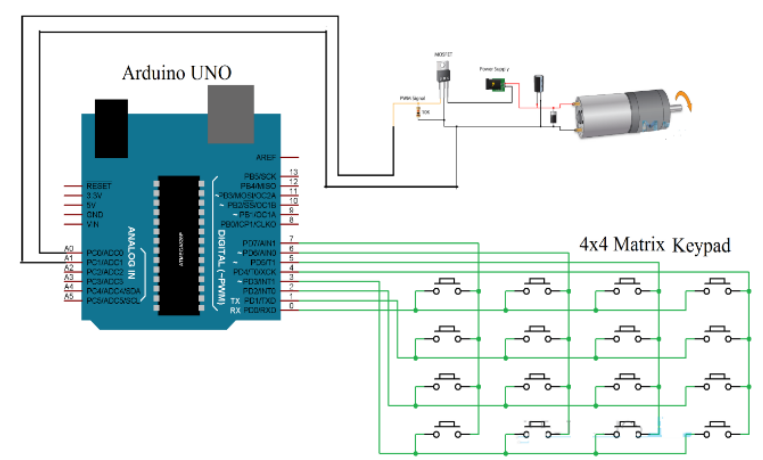

Figure 9. Prototype of proposed system with a $4 \times 4$ pressure switches and a dc motor to power elevator assembly.

\subsection{Screw Jack Input Speed,}

$\mathrm{N}(\mathrm{rpm})=\{$ Linear Speed $(\mathrm{mm} / \mathrm{min}) \times$ Gear Ratio $\} /$ $\left\{\right.$ Pitch $(\mathrm{mm}) \times \mathrm{N}^{\circ}$ of Starts on Lifting Screw

Input speed should not exceed $1800 \mathrm{rpm}$. Number of starts on lifting screw is usually 1 , unless otherwise stated.

Note: Screw Lead $=$ Pitch $\mathrm{x}$ No of Starts

\section{Operating Input Power (kW), Pin}

$$
\operatorname{Pin}(\mathrm{kW}=\{\text { Load }(\mathrm{kN}) \times \text { Linear Speed }(\mathrm{mm} / \mathrm{min})\}
$$$$
/\{60000 x \eta d\}
$$

$\eta \mathrm{d}=$ Dynamic Screw Jack Efficiency

\section{Operating Input Torque}

Tino $(\mathrm{Nm})=\{$ Pin $(\mathrm{kW}) \times 9550\} / \mathrm{N}(\mathrm{rpm})$ 
This method is very rugged, hard wired logic and efficient to save accidentally drowning victims from dangerous situations without waiting for a life-guard.

Based on the calculations, a suitable motor can be selected for the given dimensions of swimming pool.

\section{CONCLUSION}

The proposed method uses rugged and heavy-duty mechanical structures and hard-wired logic and heavy load bearing efficient models. The main duty of the swimming pool is to bring up the victim in an emergency time without compromising anything. The gravitational force, durable tactical switches, packed with powerful Arduino on-chip computer board and mechanical muscular monsters, the screw jacks together built a novel automatic drown rescue system.

\section{REFERENCES}

[1] A. Roy and K. Srinivasan, "A novel drowning detection method for the safety of Swimmers," 2018 20th National Power Systems Conference (NPSC), Tiruchirappalli, India, 2018, pp. 1-6.

[2] J. G. Ramani, J. Gayathri, R. Aswanth and M. Gunasekaran, "Automatic prevention of drowning by inflatable wristband system," 2019 5th International Conference on Advanced Computing \& Communication Systems (ICACCS), Coimbatore, India, 2019, pp. 346-349.

[3] M. A. Hayat, G. Yang, A. Iqbal, A. Saleem, A. Hussain, and M. Mateen, "The Swimmers Motion Detection Using Improved VIBE Algorithm," 2019 International Conference on Robotics and Automation in Industry (ICRAI), Rawalpindi, Pakistan, 2019, pp. 1-6.

[4] H. Liu, M. B. H. Frej and B. Wen, "A Novel Method for Recognition, Localization, and Alarming to Prevent Swimmers from Drowning," 2019 IEEE Cloud Summit, Washington, DC, USA, 2019, pp. 6571.

[5] A. Ilham et al., "AuFloat (Autonomous Float) Basedon Artificial Intelligent and LORA (Long Range) Using Haar Cascade Method for Rescuing of Water Accident Victims," 2019, International Symposium on Electronics and Smart Devices (ISESD), BadungBali, Indonesia, 2019, pp. 1-4.

[6] Y. S. K, P. O. K, and R. R. V, "Autonomous Utility Vehicle (AUVs) Based Emergency Human Drowning Detection System Using Sonar and Thermal Detection Methods," 2019 IEEE International Conference on Distributed Computing, VLSI, Electrical Circuits and Robotics (DISCOVER), Manipal, India, 2019, pp. 1-6.

[7] Muhammad Ramdhan MS1, Muhammad Ali2, et al., "An Early Drowning Detection System for the Internet of Things (IoT) Applications ", August 2018, TELKOMNIKA, Vol.16, No.4, pp. 1870 1876 ISSN: 1693-6930,
[8] A. Kulkarni, K. Lakhani, and S. Lokhande, "A sensorbased low-cost drowning detection system for human life safety," 2016 5th International Conference on Reliability, Infocom Technologies and Optimization (Trends and Future Directions) (ICRITO), Noida, 2016, pp. 301-306.

[9] Y. Nishida, K. Hiratsuka, and H. Mizoguchi, "Prototype of Infant Drowning Prevention System at Home with Wireless Accelerometer," SENSORS, 2007 IEEE, Atlanta, GA, 2007, pp. 1209-1212.

[10] CAI Xiaoyang, W. Chen and F. Lei, "Application of Image Restoration Based on Robust Estimation in Drowning Warning System," Second Workshop on Digital Media and its Application in Museum \& Heritages (DMAMH 2007), Chongqing, 2007, pp. 33-35.

[11] L. Fei, W. Xueli and C. Dongsheng, "Drowning Detection Based on Background Subtraction," 2009 International Conference on Embedded Software and Systems, Zhejiang, 2009, pp. 341-343.

[12] Soren Bonderup, Jonas Olsson, "Preventing Drowning Accidents Using Thermal Cameras", 2016, International Symposium on Visual Computing, Advances in Visual Computing pp 111122. Part of the Lecture Notes in Computer Science book series (LNCS, volume 10073).

[13] ://en.wikipedia.org/wiki/Pool_safety_camera

[14] S. Sindhuja and P. M. T. Joe, "MEMS based-self regulating airbag drowning aversion system for submerged swimmers," 2015 International Conference on Circuits, Power and Computing Technologies [ICCPCT-2015], Nagercoil, 2015, pp. $1-4$.

[15] Wenmiao Lu and Yap-Peng Tan, "Swimmer motion analysis with application to drowning detection," 2002 IEEE International Symposium on Circuits and Systems. Proceedings (Cat. No.02CH37353), Phoenix-Scottsdale, AZ, USA, 2002, pp. II-II.

[16] H. Eng, K. Toh, W. Yau, and J. Wang, "DEWS: A Live Visual Surveillance System for Early Drowning Detection at Pool," in IEEE Transactions on Circuits and Systems for Video Technology, vol. 18, no. 2, pp. 196-210, Feb. 2008.

[17] https://www.nsc.org/home-safety/tools-resources/ seasonal-safety/drowning

[18] [http://www.powerjacks.com/perch/resources/broch ure/pj-sjb-e-bs-en-01b-lowres.pdf]

[19] Pillalamarri Laxman, Dr.Anuj Jain, "A State of The Art of Drowning Death Detection and Prevention Systems with Various Communication Technologies and Discussion on their Performance", Conference icsmec2020 29th \& 30th June 2020. 